\title{
A Novel Architecture for All-Optical Add-Drop Multiplexing of OFDM Signals
}

\author{
S. Sygletos ${ }^{(1)}$, S. J. Fabbri( ${ }^{(1,2)}$, E. Giacoumidis(1), M. Sorokina ${ }^{(1)}$, D. Marom ${ }^{(3)}$, M. F. C. Stephens ${ }^{(1)}$, D. \\ Klonidis $^{(4)}$, I. Tomkos ${ }^{(4)}$ and A. D. Ellis ${ }^{(1)}$ \\ (1) Aston Institute of Photonic Technologies, Aston University, Aston Triangle, Birmingham, UK. \\ (2) Department of Physics, University College Cork, Cork, Ireland. ${ }^{(3)}$ Hebrew University of Jerusalem, \\ Jerusalem Israel. ${ }^{(4)}$ Athens Information Technology Center, Athens, Greece \\ s.sygletos@aston.ac.uk
}

\begin{abstract}
We propose a novel architecture for all-optical add-drop multiplexing of OFDM signals. Extensive numerical simulations have been carried out to benchmark the performance of the architecture against critical design parameters.
\end{abstract}

\section{Introduction}

Reconfigurable optical add-drop multiplexers (ROADM) and cross-connects established the concept of transparent networking by enabling all-optical switching and dynamic routing of the network traffic ${ }^{1,2}$. Their switching functionality is based on the use of reconfigurable optical filters in the through path for erasing the dropped channels and creating empty spectral slots for new signals to be added. With the recent introduction of orthogonal frequency division multiplexing (OFDM) in optical transmission, where neighboring channels overlap in spectrum, conventional R-OADMs are unable to perform switching without violating the orthogonality condition required for crosstalkfree performance.

Recently, Winzer in [3] proposed an optoelectronic interferometer structure to facilitate sub-channel switching within OFDM bands, through coherent subtraction and addition of the sub-carriers. This scheme, however, deviates from the traditional concept of all-optical bypassing as it requires coherent detection and electronic de-multiplexing of the whole OFDM signal and the implementation of advanced digital pulse shaping to replicate the waveform of the sub-channel that needs to be removed from the super-channel. As a result, the scheme is not scalable to high superchannel capacities (e.g. > 400Gbit/s).

In this paper, we present a novel interferometric approach to perform add-drop multiplexing of OFDM signals in the optical domain without use of optoelectronic conversion and digital signal processing. On the contrary, based on purely all-optical methods, such as FFT/i-FFT filtering ${ }^{4}$ and time domain sampling, we create a replica of the sub-channel waveform and achieve its removal from the OFDM superchannel. In depth numerical study of its performance has identified the main sources of degradation and optimized critical subsystem parameters.

\section{Proposed Scheme}

The proposed architecture is depicted in Fig. 1a). Two flexible Wavelength Selective Switch units (WSSs), one at the input and one at the output of the node, perform the super-channel selection and re-insertion back to the network traffic. Sub-channel switching is achieved by means of a three branch interferometer structure (branch -A, -B, -C). Each interferometer is capable of single super-channel processing, however, multiple sub-channels can be added or dropped within it at the same time. At the interferometer, a portion of the selected superchannel is dropped to the local receivers and the rest is split between the two branches -A and -B. Sub-channel blocking is facilitated by replicating the corresponding signal waveform and interfering it destructively with the super-channel that propagates at branch-A. This is a purely alloptical process taking place in three stages. Initially, the OFDM signal is de-multiplexed by an optical Fast-Fourier Transform processor. Subsequently, a bank of optical gates performs time sampling of the sub-channels that need to be blocked from the through path. The optical gates are synchronized to a common clock signal, extracted from the super-channel. Fig. 1b) depicts the simulated optical spectrum of an OFDM signal consisting of 7 BPSK modulated sub-carriers at $10 \mathrm{Gbit} / \mathrm{s}$, after the WSS selection. The optical spectrum of the middle (ch 4) sub-channel after the de-multiplexing with an optical FFT processor ${ }^{4}$ is also depicted. The eye diagram of ch 4, see Fig. 1-c), has reduced opening due to the preceded matched filtering and the inter-symbol interference (ISI) from the neighboring channels. In this example, crosstalk free regions cannot be identified, as the orthogonality condition has been violated due to the tight pass-band selection of the WSS ( $3^{\text {rd }}$ order Gaussian, $100 \mathrm{GHz}$ bandwidth) and the finite bandwidth of the transmitter.

The optical gate selects a window of minimum crosstalk, see Fig. 1-d), and feeds the 


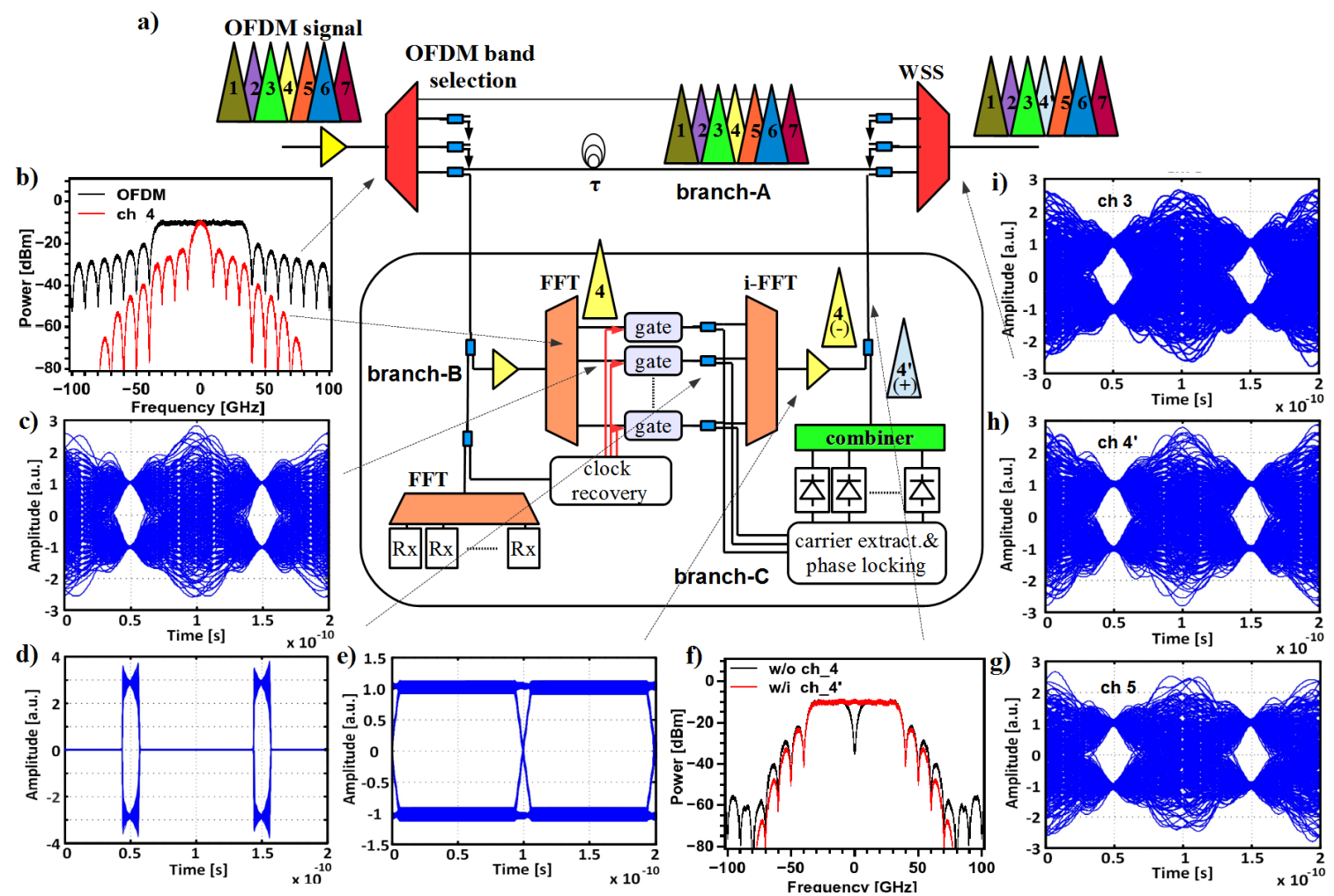

Fig. 1 : a) Diagram of proposed optical add-drop multiplexer for OFDM signals; b) Optical spectrum of input OFDM superchannel (black), and of middle sub-channel after the FFT de-multiplexing (red); c) corresponding eye-diagram of demultiplexed signal; d) eye-diagram of the gated signal; e) after the reshaping of the i-FFT filter; f) optical spectrum of the OFDM super-channel at the output of the node when the middle channel (ch 4) has been removed (black) and a new one has taken its places (red) (ch4'); g) eye diagram of ch 5 at the output of the node, h) eye diagram of ch $4^{\prime}$ at the node; i) eye diagram of ch 3 at the output of the node.

sampled waveform to an optical i-FFT processor, with transfer function $H(f)=\operatorname{sinc}(f T)$, which reshapes the pulses back to their initial symbol duration $T$, see Fig. 1-e). Finally, the recovered waveform is amplified and interfered destructively with the OFDM signal in the upper branch creating a free spectral position for a new channel to be added, see Fig. 1-f). The insertion of new channels takes place on a separate branch (i.e. branch -C), with a bank of laser transmitters aligned to the sub-channel frequencies of the OFDM super-channel. For the alignment known optical carrier extraction and phase locking methods can be applied5. In this study an ideal phase locking process has been assumed. The resulting optical spectrum, shown in Fig. 1-f), and the clear eye diagrams of the added channel (see Fig. 1-h)) and of its closest neighbors, i.e. ch 5 and ch 3 shown in Fig.1-g), Fig. 1-i), confirm that successful add-drop operation with low penalty.

\section{Results}

A single sub-channel add-drop scenario has been considered. The seven sub-carriers of the OFDM signal (see Fig. 1-a) were independently modulated using electrical pulse-streams of randomly generated data patterns and $10 \mathrm{psec}$ rise-time. The inter-channel crosstalk due to the non-rectangular pulse shaping was suppressed by setting the phase difference of the adjacent sub-carriers equal to $\pi / 2$. The system level evaluation has been achieved by direct error counting following an iterative Monte-Carlo method, which provides BER estimation with less than $10 \%$ relative error. The impact of each degradation was assessed by calculating the corresponding penalty in the received sensitivity (defined at BER $=10^{-4}$ ) at the output of the node with respect to the back-to-back performance. The simulation bandwidth was $1.28 \mathrm{THz}$.

Fig. $2(a, b)$ shows the results of the gating optimization. For these results the ASE noise of the amplifiers in the node has been ignored. In Fig. 2a) the sensitivity penalty has been characterized as a function of the switching window for ch4' and its two neighbors (ch 3 and ch 5). Ch 4' is affected mostly since it experiences the in-band crosstalk from a nonoptimized gating operation. The penalty remains below $0.2 \mathrm{~dB}$ for the three channels when the switching window is kept below 17 psec. For longer durations, the penalty for ch 4 ' is rapidly increasing $(>1.2 \mathrm{~dB})$ whereas for the other two channels, which bypass the node it does not 

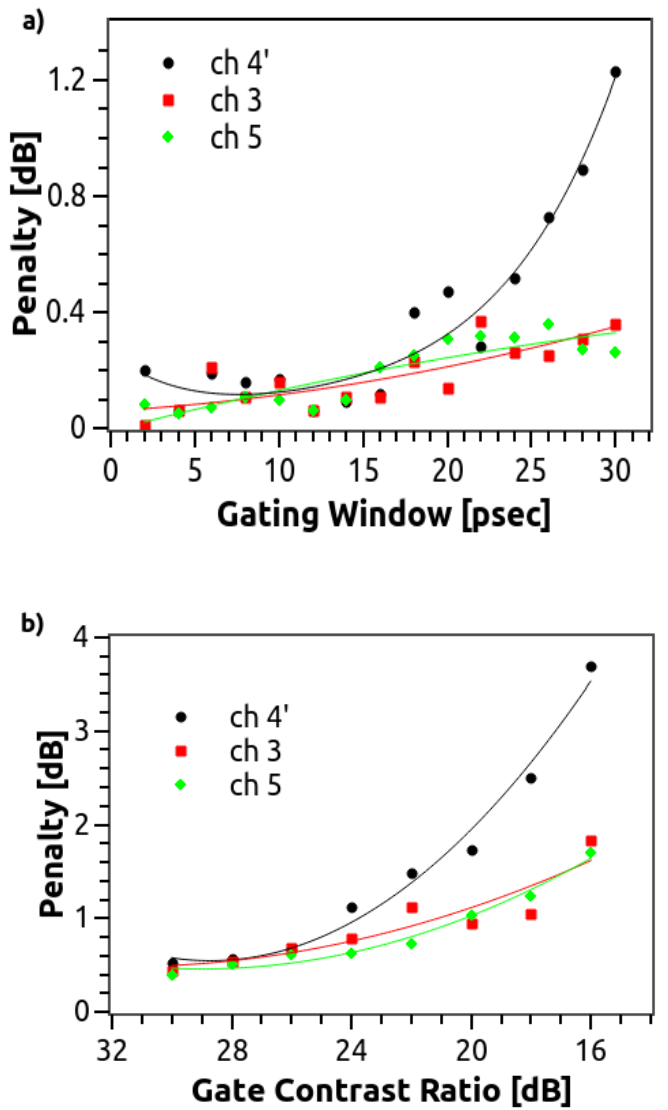

Fig. 2 Penalty for the added sub-channel (ch 4') and its two neighbours as a function of a) the switching window duration and $b$ ) the contrast ratio of the optical gate

exceed $0.3 \mathrm{~dB}$.

For the above calculations, the gate had an infinite contrast ratio, enabling complete suppression of the waveform crosstalk outside the sampling window. In real implementations the contrast ratio will be limited introducing additional degradations in the system performance. It is therefore critical to identify the specifications of the actual device for the node application. Fig. 2b) depicts the sensitivity penalty for the three channels under study (ch4', ch 3 and ch 5) as a function of the gate contrast ratio. Compared to the results of the previous figure the impact here is more detrimental. The contrast ratio should be higher than $28 \mathrm{~dB}$ to ensure penalty less than $0.6 \mathrm{~dB}$. The gating window in this case was 12 psec.

The gating action combined with the i-FFT induced pulse shaping introduces additional losses in branch-B which scale proportionally to $\sim \alpha^{2}$, where $\alpha$ is the duty cycle of the gate. These are compensated by the corresponding amplifiers (see Fig. 1a) but at the expense of a degradation in the effective noise figure. We investigated the impact of this effect by calculating the sensitivity penalty for the switched channel (i.e. ch4') as a function of the duration of the gating window for different input

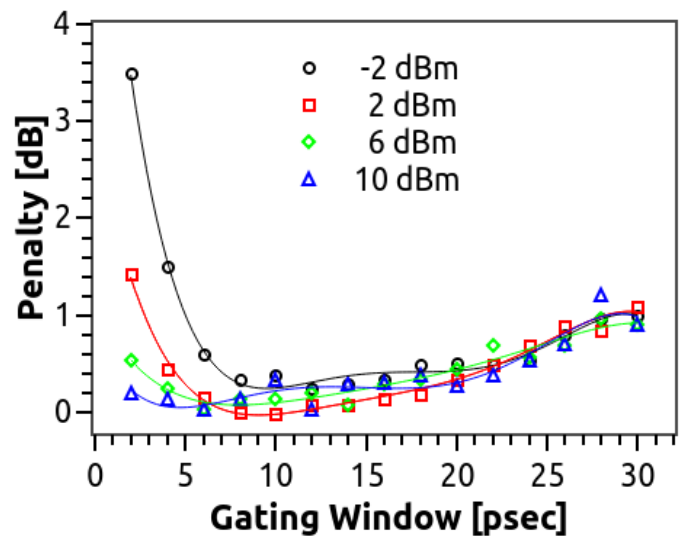

Fig. 3 Sensitivity penalty of ch $4^{\prime}$ as a function of the switching window duration for different power levels at the input of the gate

powers at the input of the gate (see Fig. 3) The noise figure of the amplifiers was set to $5 \mathrm{~dB}$ (e.g. EDFAs) and the loss of each critical building element (i.e. FFT/i-FFT filters, WSSs and gating device) to $6 \mathrm{~dB}$. The contrast ratio was set above $30 \mathrm{~dB}$. Penalty less than $0.5 \mathrm{~dB}$ is calculated when the gating window is $\sim 10$ psec. For shorter durations the penalty increases rapidly when the input power to the gate is below $6 \mathrm{dBm}$. On the contrary, for higher power levels the effective noise figure in the amplifier chain of branch-B has been improved and the performance penalty does not exceed $1 \mathrm{~dB}$.

\section{Conclusions}

A novel optical add-drop architecture for OFDM signals has been proposed which makes use of all-optical processes such as FFT/i-FFT filtering and time-domain sampling to achieve subchannel switching. In depth numerical investigation of its performance has indicated switching windows of $\sim 10$ psec and contrast ratios $>30 \mathrm{~dB}$ for optimum performance.

\section{Acknowledgements}

This work has been supported by the EU-ICT project FOX-C, the Marie Curie -IEF project ARTISTE (IEF 330697) and the Royal Society (WM120035-TEST)

\section{References}

[1] M. Feuer et al.,"ROADMs and their system applications," Ch. 8 in Optical Fiber Telecommunications VB, L.P. Kaminov, T. Li, A. Willner, Academic Press (2008)

[2] M. Vasilyev et al."Ultra-long-haul DWDM networks with Broadcast\&Select OADMs/OXCs," IEEE J. Lightw. Technol. Vol. 21, no. 11 pp 2661-2672, 2003

[3] P. Winzer, "An opto-electronic interferometer and its use in subcarier add/drop multiplexing," IEEE J. Lightw. Technol. Vol. 31, no. 11 pp 1775-1782, 2013

[4] D. Hillerkuss, et al. "Simple all-optical FFT scheme enabling Tbit/s real time signal processing" Optics Express, vol. 18, no. 9 pp. 9324-9340, (2010)

[5] L. G. Kazovsky, "Balanced phase-locked loops for optical homodyne receivers: Performance analysis, design considerations and laser linewidth requirements," J. Light. Technol., vol. 25, no. 5 March 2008 